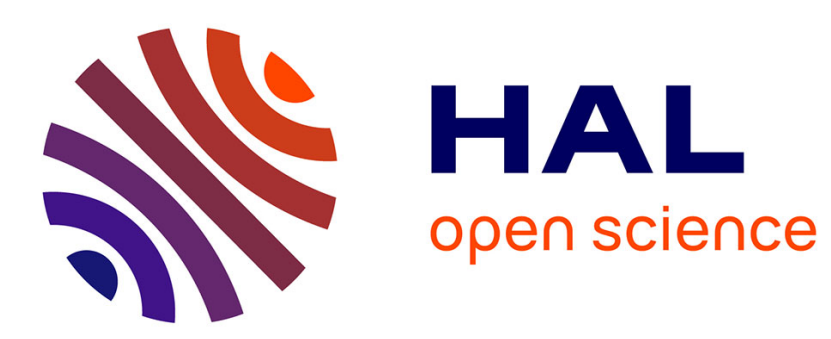

\title{
INFLUENCE DES CONDITIONS DE RECRISTALLISATION SUR LE FROTTEMENT INTÉRIEUR DU MAGNÉSIUM
}

Gilbert Fantozzi, C. Esnouf

\section{- To cite this version:}

Gilbert Fantozzi, C. Esnouf. INFLUENCE DES CONDITIONS DE RECRISTALLISATION SUR LE FROTTEMENT INTÉRIEUR DU MAGNÉSIUM. Journal de Physique Colloques, 1983, 44 (C9), pp.C9-679-C9-684. 10.1051/jphyscol:19839102 • jpa-00223335

HAL Id: jpa-00223335

https://hal.science/jpa-00223335

Submitted on 1 Jan 1983

HAL is a multi-disciplinary open access archive for the deposit and dissemination of scientific research documents, whether they are published or not. The documents may come from teaching and research institutions in France or abroad, or from public or private research centers.
L'archive ouverte pluridisciplinaire HAL, est destinée au dépôt et à la diffusion de documents scientifiques de niveau recherche, publiés ou non, émanant des établissements d'enseignement et de recherche français ou étrangers, des laboratoires publics ou privés. 


\title{
INFLUENCE DES CONDITIONS DE RECRISTALLISATION SUR LE FROTTEMENT INTÉRIEUR DU MAGNÉSIUM
}

\author{
G. Fantozzi et C. Esnouf \\ Groupe d'Etudes de Métallurgie Physique et de Physique des Matériaux*, \\ I.N.S.A., Bät. 502, 69621 Vizzeurbanne Cedex, France
}

\begin{abstract}
Résumé - Hous avons êtudié l'effet des conditions de recristallisation avant déformation plastique sur les manifestations anëlastiques d'un magnésium. de haute pureté. Nous nous sommes particulièrement intēressés à là relaxation de Bordoni et au frottement intērieur basse température.

Abstract - The effect of recrystallization before plastic cieformation on tine anelastic behaviour of a high purity magnesium has been studied. In particular, we have considered the Boraioni relaxation and the low temperature internal friction.
\end{abstract}

\section{INTRODUCTION}

Nous avons montré récemment que le spectre de frottement intérieur d'un magnésium de haute pureté déformé plastiquement est tout-à-fait similaire à celui des métaux c.f.c. $(1,2)$. I1 est constitué :

d'une part de la relaxation de Bordoni qui comprend les deux pics $B_{1}$ (situé vers $40 \mathrm{~K}$ à $1 \mathrm{~Hz}$ ) et $\mathrm{B}_{2}$ (situé vers $80 \mathrm{~K}$ ).

d'autre part de deux pics d'intéraction dislocations-défauts ponctuels $P_{1}(105 \mathrm{~K})$ et $P_{2}(220 \mathrm{~K})$

enfin d'un pic $\pi_{1}(15 \mathrm{~K})$ et d'un pic $P_{A}(420 \mathrm{~K})$. Le pic $\pi_{1}$ a été attribué à un mécanisme d'interaction entre les décrochements géométriques et des défauts de type lacunaire (3)

Les caractéristiques de Ta relaxation de Bordoni dépendent des conditions de recristallisation avant déformation $p$ lastique. Il nous a donc paru intéressant d'étudier cet effet dans le cas du magnêsium et d'une manière plus génêràle sur l'ensemble du spectre de firottement intérieur.

\section{RESULTATS EXPER IMENTAUX}

Nous avons utilisê un magnésium de haute pureté obtenu par zone fondue par le Centre d'Etudes de Chimie Métallurgique de Vitry. Ce magnésiuri contient de l'ordre d'une p.p.m. d'impuretés.

Après filage; deux traitemients de recuit ont étê pratiqués : - recuit à $300^{\circ} \mathrm{C}$ pendant 2 heures sous atmosphère d'argon. La taille des grains obtenue est de 1 'oráre du $\mathrm{mm}$.

pendant 2 heures sous atmosphère d'argon ou d'hydrogène. La taille du grain obtenue est de 1'ordre de $2 \mathrm{~mm}$.

\footnotetext{
* Laboratoire associé au C.N.R.S. $n^{\circ} 341$
} 


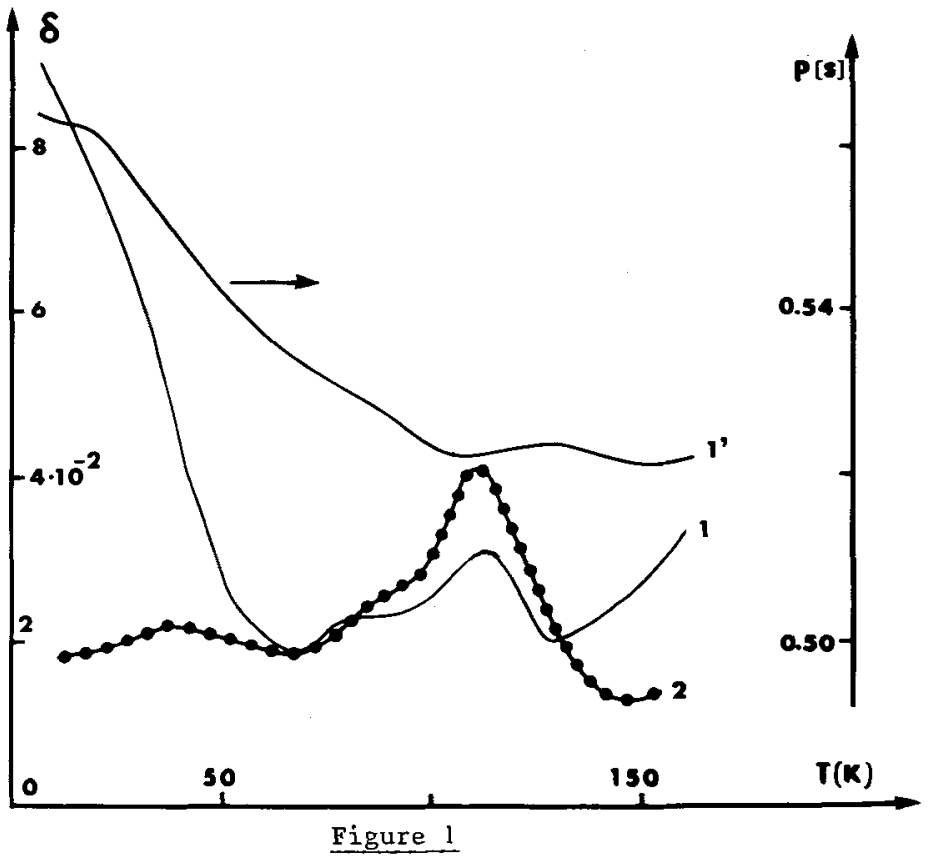

Frottement intêrieur et période après đéformation plastique de $2 \%$ à $10 \mathrm{~K}$ $d^{\prime}$ un échantillon de magnesium ; courbes $l^{\text {et }} l^{\prime}{ }^{\prime}$ : après recristallisation à $500^{\circ} \mathrm{C}$; courbe 2 : après recristallisation à $300^{\circ} \mathrm{C}\left(\varepsilon_{\mathrm{m}}=2,2 \mathrm{x} 10^{-5}\right)$.

Après recristallisation à $300^{\circ} \mathrm{C}$, les résultats obtenus ont déjà été publiês par ailleurs /1,2,4/ et nous renvoyons le lecteur aux publications citées. Après recristallisation à $500^{\circ} \mathrm{C}$ sous atmosphère d'argon, les résultats obtenus à $1^{\prime}$ 'aide d'un pendule de torsion inversé travaillant à une fréquence àe l'ordre de $1 \mathrm{hz}$ sont montrés par les figures 1 à 6 . La figure 1 présente le spectre de frottement intêrieur directement après déformation plastique par torsion de $2 \%$ à $10 \mathrm{~K}$. Il apparaît que le frottement intérieur et la période des oscillations (courbes 1 et $1^{\prime}$ ) diminuent rapidement entre 10 et $50 \mathrm{~K}$ pour $\delta$ et entre 10 et $100 \mathrm{~K}$ pour $\mathrm{la}$ période. Hous pouvons observer que cette diminution n'a pas lieu après recristallisation à $300^{\circ} \mathrm{C}$ (courbe 2 ). De plus, lors de cette première montée en température, les pics $P_{1}$ et $B_{2}$ apparaissent.

Après recuit à $150 \mathrm{~K}$, le spectre de frottement intérieur obtenu est ináiqué par la figure 2 pour deux amplitudes de mesure. Pour les faibles amplitudes de vibration, le spectre de frottement intérieur est le même qu'après recristallisation à $300^{\circ} \mathrm{C}$ : i.l est essentiellement constitué du pic de Bordoni $B_{1}$ et d'une légère composante $B_{2}$. Pour une amplitude de mesure $\varepsilon_{\mathrm{m}}=2,210^{-5}$, le fond continu beaucoup plus important augmente notablement au-dessus de $100 \mathrm{~K}$ environ alors qu'aprës recrista $1-$ lisation à $300^{\circ} \mathrm{C}$, on $n^{\prime}$ observe pas de fond continu important pour de telles amplitudes.

Les mêmes observations peuvent être faites après recuit à $250 \mathrm{~K}$ (fig. 3 ), $340 \mathrm{~K}$ (fig. 4), $400 \mathrm{~K}$ (fig. 5) et $500 \mathrm{~K}$ (fig. 6). Après recuit à $250 \mathrm{~K}$, on observe à faible amplitude les trois pics $\pi_{1}$, $B_{1}$ et $B_{2}$ mais pour l'amplitude de mesure plus élevée le fond continu est nettemênt p̧lus important, le pic $\pi_{1}$ disparait et le pic 


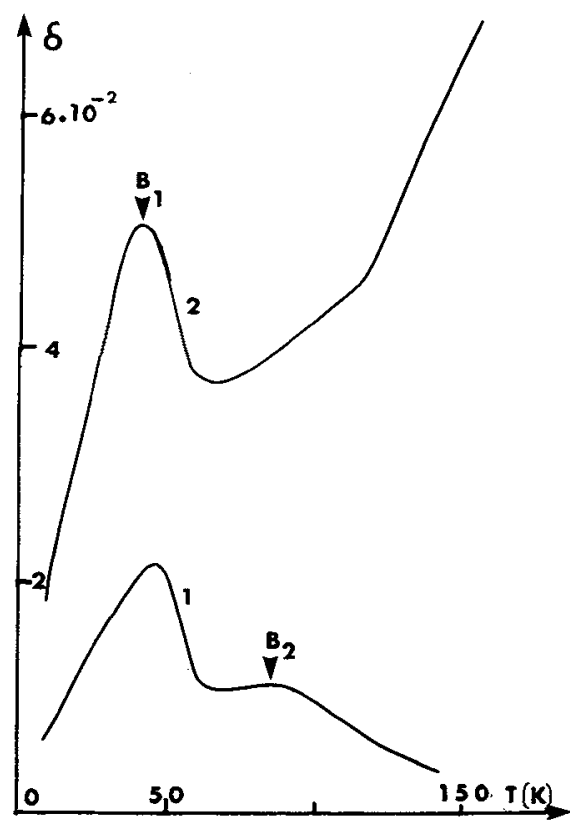

Figure 2

Frottement intérieur après déformation plastique de $2 \%$ à $10 \mathrm{~K}$ et recuit à $150 \mathrm{~K}$ courbe $1: \varepsilon_{\mathrm{m}}=4,5 \times 10^{-6}$; courbe 2 : $\varepsilon_{\mathrm{m}}=2,2 \times 10^{-5}$.

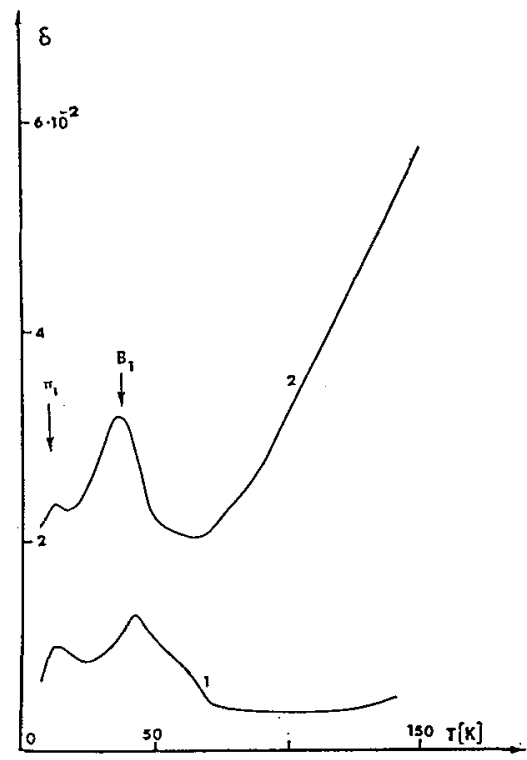

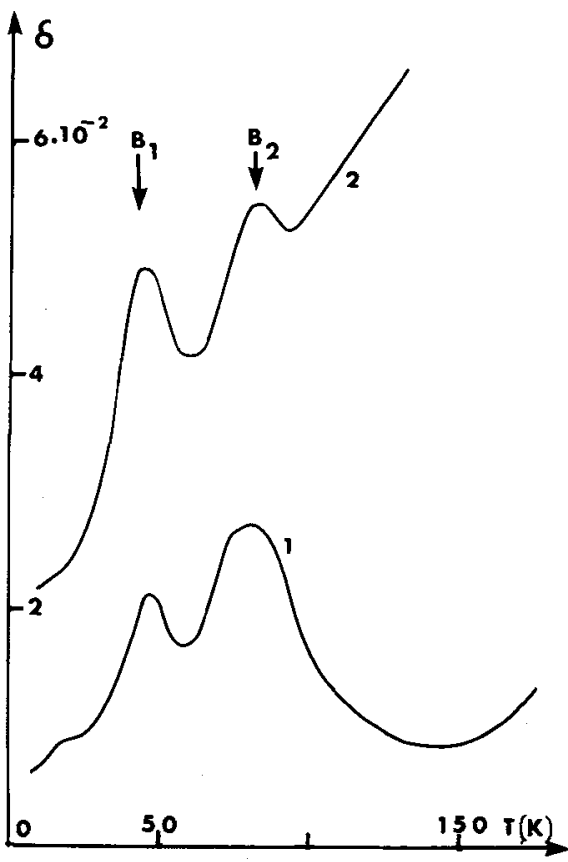

\section{Figure 3}

Frottement intérieur après déformation plastique de $2 \%$ à $10 \mathrm{~K}$ et recuit à $250 \mathrm{~K}$; courbe $1: \varepsilon_{\mathrm{m}}=4,5 \times 10^{-6}$ courbe $2: \varepsilon_{\mathrm{m}}=2,2 \times 10^{-5}$

\section{Figure 4}

Frottement intérieur après déformation plastique de $2 \%$ à $10 \mathrm{~K}$ et "recuit à $340 \mathrm{~K}$; courbe $1=\varepsilon_{\mathrm{m}}=4,5 \times 10^{-6}$; courbe $2: \varepsilon_{\mathrm{m}}=2,2 \times 10^{-5}$. 


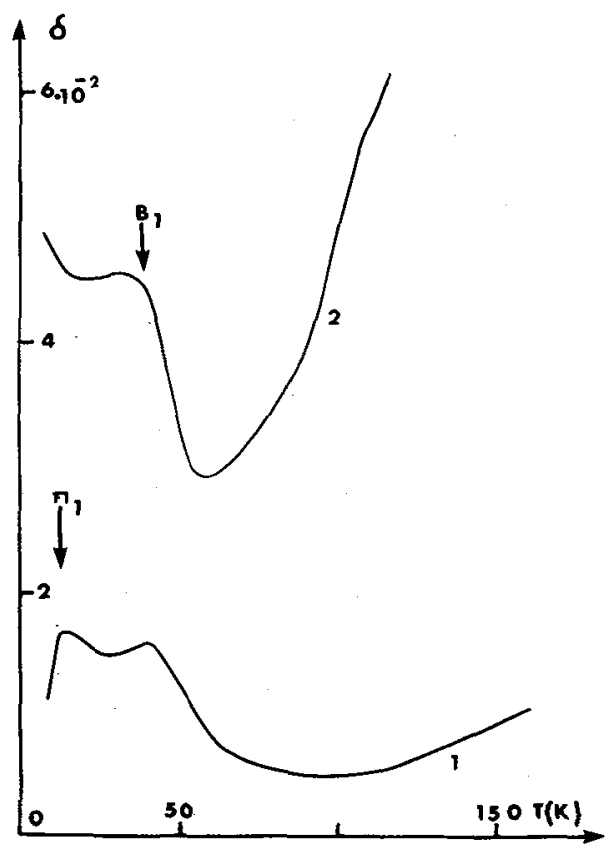

\section{Figure 5}

Frottement intêrieur après déformation plastique de $2 \%$ à $10 \mathrm{~K}$ et recuit à $400 \mathrm{~K}$; courbe $\mathrm{l}: \varepsilon_{\mathrm{m}}=4,5 \times 10^{-6}$; courbe $2: \varepsilon_{\mathrm{m}}=2,2 \times 10^{-5}$.

\section{Figure 6}

Frottement intérieur après déformation plastique de $2 \%$ à $10 \mathrm{~K}$ et recuit à $500 \mathrm{~K}$; courbe $1: \varepsilon_{\mathrm{m}}=4,5 \times 10^{-6}$; courbe $2: \varepsilon_{\mathrm{m}}=2,2 \times 10^{-5}$. 
$B_{2}$ est beaucoup moins net. Après recuit à $340 \mathrm{~K}$ et $400 \mathrm{~K}$, nous observons les deux pics $\pi_{j}$ et $B_{1}$, le pic $B_{2}$ ayant disparu. Pious pouvons remarquer que le pic de Bordoni $B_{1}$ croit avec $\varepsilon_{m}$ tandis que le pic $\pi_{1}$ diminue ou disparait quand 1 'amplitude de vibration de mesure augmente. Ceci est particulièrement net après recuit à $500 \mathrm{~K}$. Le pic $B_{1}$ est presque absent à faible amplitude alors qu'il est netteraent développé à forte amplitude de vibration.

De plus, nous pouvons remarquer que le frottement intērieur devient très êlevé à $10 \mathrm{~K}$ ă forte amplitude de vibration pour des températures de recuit supérieures à $350 \mathrm{~K}$. Après recristallisation à $500^{\circ} \mathrm{C}$ sous atmosphère d'hydrogène, le spectre de frottement intérieur observé après déformation plastique est tout-à-fait similaire a celui obtenu après recristallisation à $500^{\circ} \mathrm{C}$ sous atmosphère d'argon. Seul le pic $\pi_{1}$ disparaît même à faible amplitude de vibration.

\section{DISCUSSION}

Les résultats précêdents montrent que les conditions de recristallisation modifient le spectre de frottement intérieur après déformation plastique.

Immédiatement après déformation plastique à basse tempërațure, le frottement intérieur mesuré à $10 \mathrm{~K}$ est très important (de $7^{\prime}$ ord́re de $10^{-1}$ ) après recristallisation à $500^{\circ} \mathrm{C}$.

Ce frottement intérieur élevé à basse température est 1 'indication de l'existence d'un pic de frottement intérieur important situé entre 0 et $10 \mathrm{~K}$. I1 serait intéressant d'effectuer des mesures de frottement intērieur à très basse température pour préciser ce point. Ce frottement intérieur à basse température évolue avec la tempêrature de recuit. II commence par disparầtre puis rêapparaît après recuit vers $400 \mathrm{~K}$. Le frottement intērieur basse tempërature ne peut être attribué qu'au mouvement des décrochements géométriques. Plusieurs mécanismes de dissipation d'énergie peuvent être envisagés. Par exemple, un phénomène de frottement intérieur dû à 7 'existence de contraintes de Peierls de deuxième espèce (pic de Bordoni de deuxième espèce) peut être envisagé. On peut également penser à des phênomènes d'intéraction entre les décrochements géométriques et des dëfauts ponctuels créés par déformation plastique. Ainsi, après irradiation aux électrons, MINIER et al 15 / ont observé ?'existence de pics situés à basse température (pic $\pi$ 'o et $\pi_{0}$ situés respectivement vers 10 et $15 \mathrm{~K}$ pour des fréquences de vibration de 7 'ordre de $800 \mathrm{~Hz}$ ). Ces pics apparaissent et disparaissent dans un domaine restreint de température et ont été attribués par les auteurs à une interaction des décrochements géométriques avec des défauts ponctuels de type interstitiel. On pourrait ainsi envisager un phénomène similaire dans le cas d'une déformation plastique. Une étude plus complẽte permettrait de répondre de manière plus précise à cette question.

D'une façon générale, les résultats prêsentês précédemment montrent qu'il n'y a pas de modifications notables du spectre de frottement intérieur en particulier sur la relaxation de Bordoni quand les conditions de recristallisation diffèrent. De la même manière, une recristallisation à $500^{\circ} \mathrm{C}$ sous atmosphère d'hydrogène ne perturbe pas 1'existence des pics $B_{1}$ et $B_{2}$. Or une quantité de 1 'ordre de $100 \mathrm{ppm}$ d'hydrogène est introduite dans 7 'échantiTion par un tel traitement $/ 6 /$. Par consëquent, cette expérience montre clairement que 1 'hydrogène ne peut être responsabie de la relaxation de Bordoni. Par contre, la disparition du pic $\pi_{1}$ aprẽs traitement sous hydrogène peut être liẻe à un piégeage des défauts de type lacunaire par 1 'hydrogène.

Dans tous les cas, la recristallisation a $500^{\circ} \mathrm{C}$ fait apparâtre un fond continu très important dès $100 \mathrm{~K}$ environ pour des amplitudes de mesure élevées (de l'ordre de $\left.10^{-5}\right)$. L'importance de ce fond continu et sa grande sensibilité à 7 'amplitude de vibration sont probablement liès à une augmentation des longueurs de boucles de dislocation. En effet, le fond continu au-dessus de la température des pics de Bordoni est probablement dú à une interaction athermique dislocation-défaut ponctuel. Cette interaction décrite par les auteurs lors de la troisième conférence européenne 
/7/, est très sensible d'une part à la censité de défauts porctuels situés au voisinage du plan de glissement de la dislocation et d'autre part à la longueur des disfocations et 1 'amplitude de vibration de mesure. Ainsi le fond continu est proportionnel au cube de la longueur des dislocations, les autres parametres (densitē de dislocations et de défauts ponctuels) étant maintenus constants. La température de recristallisation joue un rôle important sur le comportement du fond continu.

Enfin, nous observons qu'après déformation plastique à $10 \mathrm{~K}$ et recuit au-dessus de $300 \mathrm{~K}$, le pic $\mathrm{B}_{1}$ apparait d'autant plus nettement que 1 'amplitude de vibration est grande. Cecì est très net aprẽs recuit à $500 \mathrm{~K}$ (fig. 6 ) et met clairement en évidence que la condition de PARE /8/ indispensable à 1 'apparition de la relaxation de Bordoni peut être remplie par la contrainte de mesure appliquée.

De plus, le pic $\pi 1$ qui apparaît après recuit au-dessus de $250 \mathrm{~K}$ est d'autant plus visible que 1 'amplitude de mesure est plus faible (cf. figures 4 à 6 ). Une étude plus précise du comportement du pic $\pi_{1}$ serait souhaitable pour essayer de prêciser les mécanismes responsables du pic $\pi_{1}$ ainsi que les phénomènes de relaxation à très basse température.

\section{REMERCIEMENTS}

Nous tenons à remercier Monsieur REVEL pour la préparatión du magnésium au C.E.C.M. de Vitry.

\section{REFERENCES}

/1/ SEYED REIHANI S.M., Thèse, Universitē de Lyon, 1981.

12/ FANTOZZI G., SEYED REIHANI S.M., ESHOUF C. et REVEL G., 3-ECIFUAS, Manchester, Ed. C.C. Smith, Pergamon Press, (1980) p. 85.

13/ FANTOZZI G., ESNOUF C., LAUZIER J., MINIER C., REVEL G., à parầtre.

14/ FANTOZZI G., ESHOUF C., SYED REIHANI S.M., REVEL G., à paraître.

/5/ MINIER C., DUCLOS D., LAUZIER J., J Phys. F : Met. Phys., 13 (1983) 13.

16/ TZANETAKIS, Thèse, Universitē de Grenoble, 1978.

/7/ ESNOUF C., FARTOZZI G., 3-ECIFUAS, Manchester, Ed. C.C. Smith, Pergamon Press (1980) p. 103.

18/ FANTOZZI G., ESNOUF C., BENOIT W., RITCHIE I., Prog. Mat. Sci., 27 (1982) 311. 